\title{
Onset and severity of hearing loss due to congenital rubella infection
}

\author{
N J WILD, S SHEPPARD, R W SMITHELLS, H HOLZEL, ${ }^{*}$ AND G JONES* \\ University Department of Paediatrics and Child Health, The General Infirmary at Leeds and *Department of \\ Microbiology, Hospital for Sick Children, London
}

SUMMARY Of 111 children born in 1978-82 with congenital rubella infection, confirmed by the detection of rubella specific IgM shortly after birth, 68 were reported to be hearing impaired when notified to the National Congenital Rubella Surveillance Programme (NCRSP). The average age at which the diagnosis of hearing impairment was confirmed was 11.6 months. Recent audiograms showed a hearing loss that was usually uniform across all frequencies, equal in both ears and severe, averaging $93 \mathrm{~dB}$ across the range $250-4000 \mathrm{~Hz}$. Despite the early diagnosis of congenital rubella and the known risk of deafness, only eight of 57 infants had been tested for auditory evoked responses in the first 6 months of life; all eight had severe hearing loss. Evidence of progressive hearing loss was found in only one of the 57 children. Definitive hearing tests (as distinct from screening tests) were frequently delayed until after the first birthday. Newborn babies identified as being at high risk of congenital deafness should have a full audiological assessment in early infancy.

Rubella infection acquired during early pregnancy may cause congenital defects, of which sensorineural deafness is by far the most common and often occurs in isolation. ${ }^{1}$ Although the hearing loss is usually identified in early childhood, there have been reports of children with a progressive auditory impairment beginning several years after birth. ${ }^{2}$ The diagnosis of congenital rubella in some studies was based on clinical grounds alone ${ }^{3}$ however, and in others on the presence of IgG antibodies to rubella that could have been acquired postnatally. ${ }^{4}$ It is therefore possible that in some cases deafness was not caused by intrauterine rubella infection but was due to some other cause, for example, autosomal recessive inheritance. ${ }^{5}$

Laboratory tests for rubella specific IgM allow a more accurate diagnosis of congenital rubella infection to be made in newborn babies who have been exposed to the virus in utero. ${ }^{6}$ If the infection occurs before the 18th week of pregnancy there is a high risk of sensorineural hearing loss, ${ }^{1}$ even if the child appears unaffected at birth. A conductive loss may be superimposed in later childhood. ${ }^{1}$

Early detection and treatment of severe hearing impairment has been shown to result in a much better long term functional outcome. ${ }^{7}$ Knowledge of the onset, severity, and progression of hearing loss in children with confirmed congenital rubella infection would indicate the appropriate timing of hearing tests and duration of follow up. A retrospective review of the progress of deafness in a large cohort of children with congenital rubella that had been proved in the laboratory was therefore undertaken. In addition to providing information about the natural history of this disorder, the effectiveness of the services involved in the detection of hearing loss in early childhood has been assessed.

\section{Methods}

The records of the National Congenital Rubella Surveillance Programme (NCRSP) were examined and 117 children born in the five years 1978 to 1982 in whom congenital rubella was diagnosed by the detection of rubella specific IgM in early infancy were identified. The physician who notified each child was contacted and asked to provide further information. For children known to have a hearing loss, this included the age at which it was first suspected, by whom, and the age at which it was confirmed. For those children believed to have normal hearing or for whom this information was not available at the time of notification we attempted to determine the normality or otherwise of hearing. 
The timing and results of past hearing tests were obtained for children with a hearing loss, and wherever possible a recent pure tone audiogram was obtained for all children in the study.

\section{Results}

Of the 117 children identified, five had died and one had emigrated; these children were excluded from further analysis. Of the remaining 111 children, 68 $(61 \%)$ were recorded as having a hearing loss, 37 were initially reported to have normal hearing, and six had not had accurate testing because of developmental delay (table).

Replies to the request for details of the age at which a hearing loss was first suspected, and then confirmed, were obtained for 57 of the 68 children reported to have a hearing loss. The mean age at which the hearing loss was suspected and confirmed were 7.9 months and 11.6 months respectively. Parents were usually the first to suspect a hearing deficit (22 cases), but paediatricians (14 cases), health visitors (seven cases), audiology and ear, nose, and throat clinics (four cases), and clinical medical officers (three cases) also identified previously unsuspected cases.

In the first six months of life only eight of these children were tested for auditory evoked responses and in each case these were abnormal. Unfortunately it was not possible to determine retrospectively whether these children were tested because of their high risk of deafness or because of clinical suspicion of a hearing loss. In the second six months, 24 children had their hearing loss diagnosed by distraction tests. Six children had been reported as having normal hearing when tested by this method, however, and it is likely that a number of those whose

Table Audiological follow up of confirmed cases of congenital rubella $(n=117)^{*}$

\begin{tabular}{lc}
\hline \multicolumn{1}{c}{$\begin{array}{c}\text { Initially reported deaf }(n=68) \\
\text { Audiogram not obtained }\end{array}$} & 9 \\
Severe mental retardation & 5 \\
Hearing loss—best point for the better ear $(\mathrm{dB}):$ & 2 \\
$<40$ & 13 \\
$40-60$ & 13 \\
$61-80$ & 17 \\
$81-100$ & 9 \\
$>100$ & \\
\multicolumn{1}{l}{ Initially reported normal, or not tested $(n=43)$} \\
Audiogram not obtained & 11 \\
Normal hearing & 19 \\
Unilateral loss & 3 \\
Bilateral loss & 10 \\
\hline
\end{tabular}

${ }^{*}$ Five children died and one emigrated. hearing loss was detected after the first year also 'passed' a distraction screening test in infancy.

A significant hearing loss had been confirmed by the end of the first year in 37 cases, and by the age of 18 months in a further 13 children. None of these children showed any evidence of deterioration in hearing after the diagnosis had been established. Of the remaining seven children, six were identified as hearing impaired between 22 and 42 months (the age was unknown in one case). One of these children had a clearly documented deterioration over a five year period after initially appearing to have normal hearing. The reasons for delay in diagnosis in the other five were: failure to do appropriate tests $(n=3$, in two cases because the child had passed a distraction test), failure to attend follow up appointments $(n=1)$, and difficulty in evaluating a moderate mixed hearing loss $(n=1)$. There was no evidence of a progressive hearing loss in any of these children.

A recent pure tone audiogram was obtained for 50 of the 68 children with a known hearing loss at a mean age of 6.9 years (range 3.5 to 8.5 ). The best point for the better ear on these audiograms was between $40-60 \mathrm{~dB}$ for 13 children, $61-80 \mathrm{~dB}$ for 13 children, and 81-100 dB for 17 children. A further five children had no hearing below $101 \mathrm{~dB}$ and only two had hearing at better than $40 \mathrm{~dB}$ loss. The mean best point for the better ear was $76.0 \mathrm{~dB}$ for the whole group.

There did not appear to be a characteristic pattern of hearing loss such as high tone deafness, but the loss was remarkably symmetrical in many cases, the maximum difference between the two ears averaging only $22 \mathrm{~dB}$. The hearing deficit was usually severe with an average loss over the range $250-4000 \mathrm{~Hz}$ of $95 \mathrm{~dB}$ in the left ear and $92 \mathrm{~dB}$ in the right ear (figure). Pure tone audiograms were not obtained for 18 children of whom four had severe mental handicap and hearing loss, four had unmeasurable hearing levels, and 10 were not available for testing.

Of the $\mathbf{4 3}$ children initially reported to the NCRSP as having normal hearing or in whom testing had not been carried out, 15 subsequently had normal pure tone audiograms and a further four were reported to have normal hearing. Maternal rubella infection occurred at 20 weeks' gestation or later in four of the 15 cases with normal audiograms. Three children had a unilateral hearing loss which was mild in one case and moderate in the other two (one uniform loss, one high tone). Bilateral hearing loss was found in 10 children in this group and was severe in all but one. Of the children with a severe loss one had had abnormal responses to distraction testing at 11 months and a severe hearing loss had been 


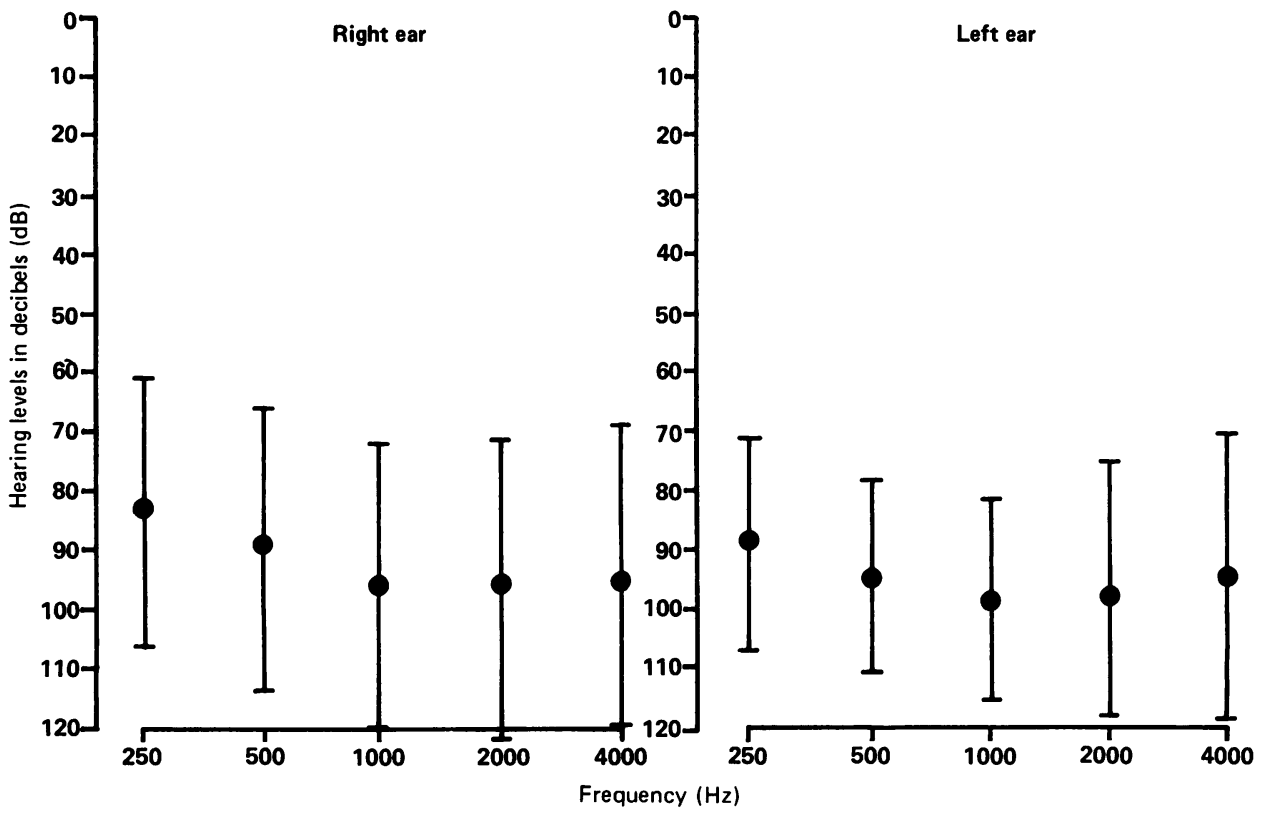

Figure Mean (SD) hearing threshold in 50 children initially reported as hearing impaired.

confirmed at 18 months. The deficit was identified by 2.5 and 3 years respectively in two others, but information relating to the onset was not available for the remaining six children with a severe loss. There was no evidence to suggest a later onset or progression in any of the deaf children who had initially been reported to have normal hearing. No information was available for 11 of the 43 children in this group.

\section{Discussion}

The data we have obtained confirm that the hearing loss that results from congenital rubella infection is severe and affects most children born to mothers who contract this infection before 18 weeks' gestation. However, the nature of the hearing loss we found is at variance with previously reported characteristics in two important respects. The first difference we found was that the hearing loss was surprisingly uniform across all frequencies and not a high tone deafness as is often stated. ${ }^{3}$ Secondly, we found little evidence to support the suggestion that the hearing loss due to congenital rubella infection is progressive. Only one patient out of a total of 61 with a bilateral loss of over $40 \mathrm{~dB}$ had a clearly documented deterioration. By contrast, all of the eight children who had auditory evoked responses in the first six months of life were found to have severe losses. As most of the children were not tested in early life, however, we cannot be certain that the hearing loss caused by congenital rubella infection is not progressive in some infants at least in the first year of life.

Almost a third of the children in this study were not diagnosed as hearing impaired until the second or third year of life. This appeared to be the result of a combination of factors that led to either a failure to investigate, or inadequate investigation of, these extremely high risk patients. The factors included: (i) a lack of awareness of, or access to, equipment to measure brain stem evoked responses, (ii) failure of physicians to appreciate the need to assess the hearing of these children in early infancy, and (iii) reluctance of some parents to accept both the possibility of a hearing disorder and the need for testing.

In summary, the hearing loss caused by congenital rubella is usually severe and affects all frequencies uniformly. Parents of newborns at risk require careful counselling, and early audiological assessment should be arranged in a specialist centre in order to allow early amplification to be provided where appropriate.

We thank the physicians who kindly provided the information and Mrs Eileen Wood for preparing the manuscript. 


\section{References}

1 Munro ND, Sheppard S, Smithells RW, Holzel H, Jones G. Temporal relations between maternal rubella and congenital defects. Lancet 1987;ii:201-4.

2 Anvar B, Mencher GT, Keet SJ. Hearing loss and congenital rubella in Atlantic Canada. Ear Hear 1984;5:340-5.

3 Sheridan MD. Final report of a prospective study of children whose mothers had rubella in early pregnancy. $\mathrm{Br}$ Med $\mathrm{J}$ 1964;ii:536-9.

4 Peckham CS. Clinical and laboratory study of children exposed in utero to maternal rubella. Arch Dis Child 1972;47:571-7.

5 Harper PS. Practical genetic counselling. Bristol: Wright, 1984.
${ }^{6}$ Cradock-Watson JE, Ridehalgh MKS. Specific immunoglobulins in children with the congenital rubella syndrome. J Hyg (Camb) 1976;76:109-23.

7 Markides A. Age at fitting of hearing aids and speech intelligibility. $\mathrm{Br} J$ Audiol 1986;20:165-7.

Correspondence to Dr NJ Wild, Department of Paediatrics and Child Health, The University of Leeds, D Floor, Clarendon Wing, The General Infirmary at Leeds, Belmont Grove, Leeds LS2 9NS.

Accepted 16 March 1989 